\title{
High prevalence of chronic kidney disease in a community survey of urban Bangladeshis: a cross-sectional study
}

\author{
Shuchi Anand ${ }^{1,3^{*}}$, Masuma Akter Khanam², ${ }^{2,4}$ Juliann Saquib', Nazmus Saquib', Tahmeed Ahmed², Dewan S Alam²,
} Mark R Cullen', Michele Barry ${ }^{1}$ and Glenn M Chertow ${ }^{1}$

\begin{abstract}
Background: The burden of chronic kidney disease (CKD) will rise in parallel with the growing prevalence of type two diabetes mellitus in South Asia but is understudied. Using a cross-sectional survey of adults living in a middle-income neighborhood of Dhaka, Bangladesh, we tested the hypothesis that the prevalence of CKD in this group would approach that of the U.S. and would be strongly associated with insulin resistance.

Methods: We enrolled 402 eligible adults (>30 years old) after performing a multi-stage random selection procedure. We administered a questionnaire, and collected fasting serum samples and urine samples. We used the Chronic Kidney Disease Epidemiology Collaboration (CKD-EPI) equation to estimate glomerular filtration rate, and sex-specific cut offs for albuminuria: $>1.9 \mathrm{mg} / \mathrm{mmol}(17 \mathrm{mg} / \mathrm{g})$ for men, and $>2.8 \mathrm{mg} / \mathrm{mmol}(25 \mathrm{mg} / \mathrm{g})$ for women. We assessed health-related quality of life using the Medical Outcomes Study Short Form-12 (SF-12).

Results: A total of 357 (89\%) participants with serum samples comprised the analytic cohort. Mean age of was 49.5 ( $\pm 12 \cdot 7)$ years. Chronic kidney disease was evident in 94 (26\%). Of the participants with CKD, 58 (62\%) had albuminuria only. A participant with insulin resistance had a 3.6 -fold increase in odds of CKD (95\% confidence interval 2.1 to 64). Participants with stage three or more advanced CKD reported a decrement in the Physical Health Composite score of the SF-12, compared with participants without CKD.
\end{abstract}

Conclusion: We found an alarmingly high prevalence of CKD—-particularly CKD associated with insulin resistance-in middle-income, urban Bangladeshis.

Keywords: Chronic kidney disease, Albuminuria, Insulin resistance, South Asia, Low-income countries

\section{Background}

Until recently, most experts presumed that glomerulonephritis or interstitial nephritis were predominant reasons for chronic kidney disease (CKD) in low-and middle-income regions including South Asia [1]. But with an aging population and rapid urbanization, obesity and type two diabetes mellitus (T2DM) afflicts a growing proportion of adults [2,3]. In fact, T2DM already affects nearly $10 \%$ of the South Asian population, a burden that approaches that of the U.S., and one that will

\footnotetext{
* Correspondence: sanand2@stanford.edu

'Stanford University School of Medicine, 291 Campus Dr, Stanford, CA 94305, USA

${ }^{3}$ Division of Nephrology, Stanford University School of Medicine, 777 Welch Road, Suite DE, Palo Alto, CA 94304, USA

Full list of author information is available at the end of the article
}

likely rise at a much faster rate over the next two decades [4]. The prevalence of CKD, particularly CKD related to insulin resistance, will rise in parallel but has been understudied.

Few [5-7] studies have examined the prevalence of CKD in South Asia: most have not used a standard definition of CKD, nor have they assessed albuminuria. In addition, the populations surveyed have not been those at highest risk (i.e., middle income, urban adults who have greater access to energy-dense processed or fast foods). Urban adults have also adopted a sedentary lifestyle, owing in part to the changing nature of their employment, crowding in residential areas, and the ubiquity of televisions [8].

\section{Biomed Central}

(c) 2014 Anand et al.; licensee BioMed Central Ltd. This is an Open Access article distributed under the terms of the Creative Commons Attribution License (http://creativecommons.org/licenses/by/2.0), which permits unrestricted use, distribution, and reproduction in any medium, provided the original work is properly credited. 
To determine the prevalence of chronic diseases in a high-risk group, we performed a cross-sectional multistage random sampled survey of adults living in a middle-income neighborhood in Dhaka, Bangladesh. We hypothesized that the prevalence of CKD would approach that of the U.S., and that a majority of participants with CKD would possess features of insulin resistance.

\section{Methods}

We performed a cross-sectional survey of 402 adult residents of Dhaka to examine the prevalence of obesity and chronic diseases, including T2DM, hypertension, and CKD. This multistage cluster random sampled survey consisted of: 1 . a patient questionnaire, 2. anthropometric measurements, and 3. fasting serum sample and urine collection. The Institutional Review Board at Stanford University and the Ethical Review Committee at International Center for Diarrheal Diseases Research, Bangladesh (ICDDR,B) approved the study.

\section{Survey design}

We obtained a detailed map of Mohammedpur, an urban residential area organized in the 1950s within Dhaka with a population of about 450,000 people living in 100,000 households [9]. Of the six blocks that comprise the district, we randomly selected three. We then assigned a study identification number to all of the buildings in the three blocks, and used random sample generator software (SPSS, Chicago, IL) to create a list of 500 buildings. We selected extra buildings to ensure enrollment of one person per building. Most buildings in Mohammedpur are apartment complexes. On arrival at a selected building, the survey team-made up of a research assistant, study nurse and study supervisor-counted the total number of households, and utilized a random number generator to select a single household. If the household members declined to participate or there was non-response, the survey team randomly selected another household within the same building.

Eligible participants were at least 30 years old; if there were more than one eligible participant in the selected household, we randomly selected one from all eligible participants using the same random number generator. We targeted equal enrollment of men and women in the 30 to 50 and $>50$ year age category (i.e., 100 individuals in each category). We enrolled participants until the quota for any given combination was reached, after which participants were enrolled only into the remaining strata.

\section{Data collection}

After obtaining informed consent, research assistants administered a patient questionnaire in Bangla. Using a structured questionnaire, we obtained data on: socioeconomic status including education and occupation, tobacco use, self-reported personal and family history of chronic diseases, diet and physical activity, and family planning. We administered the Medical Outcomes Study Short-Form-12 version two questionnaire (SF-12) to assess health related quality of life (HRQoL). At the completion of the patient questionnaire, the research assistant made an appointment for a second visit (within one week) to obtain a urine specimen, fasting serum sample, and anthropometric measurements.

A study nurse measured height, weight, and waist and hip circumference on all participants using standardized procedures [10]. The nurse also obtained two blood pressure readings using a manual sphygmomanometer after the participant had remained in a seated position for five minutes with his or her arm resting on the chair. The nurse used standard phlebotomy procedures to obtain a fasting serum sample and a urine specimen.

\section{Laboratory analyses}

We transported serum and urine samples to the ICDDR, B central laboratory within one hour of collection. We processed all samples for serum creatinine, hemoglobin A1c, and fasting glucose. Due to budgetary limitations, we performed more expensive tests - urine albumin and creatinine, and serum lipids and triglycerides - in a select group of participants. This group $(n=341)$ fit at least one criteria for metabolic syndrome, had urine dipstick positive for proteinuria, or had risk factors for CKD (i.e., self-reported personal or family history of diabetes, hypertension, or CKD, or self-reported personal history of auto-immune disease, kidney stones, or birth weight below $2.5 \mathrm{~kg}$ ) [11].

Urine albumin was measured by immunoturbidimetric assay using Olympus AU 640, and plasma and urinary creatinine were measured by enzymatic colorimetric assay using Olympus AU 640 (Olympus corporation, Tokyo, Japan). Assay variation was checked daily with an internal quality assessment scheme, and the coefficient of variation for each test was below $5 \%$.

\section{Definitions of chronic diseases and risk factors}

We defined a participant as having T2DM if he or she had fasting blood glucose $\geq 7.0 \mathrm{mmol} / \mathrm{L}$ or hemoglobin A1c $\geq 6.5 \%$ or medication use for self-reported diabetes. We defined metabolic syndrome according to International Diabetes Federation criteria [12] as centripetal obesity (waist circumference $\geq 90 \mathrm{~cm}$ for men and $\geq 80 \mathrm{~cm}$ for women) plus any two of the followings: (1) triglycerides $>17 \mathrm{mmol} / \mathrm{L}$ or treatment for elevated triglycerides, (2) HDL cholesterol $<1.03 \mathrm{mmol} / \mathrm{L}$ in men or $<129 \mathrm{mmol} / \mathrm{L}$ in women, or treatment for low HDL, (3) systolic blood pressure $>130 \mathrm{mmHg}$, diastolic blood pressure $>85 \mathrm{mmHg}$, or treatment for hypertension, and (4) fasting plasma glucose $>5.6 \mathrm{mmol} / \mathrm{L}$. We defined a 
participant as having insulin resistance if he or she met criteria for T2DM or metabolic syndrome. We used the following categories for Quételet's (body mass) index (BMI): $<25$ (lean or normal), 25 to $<30$ (overweight), and $\geq 30 \mathrm{~kg} / \mathrm{m}^{2}$ (obese). We captured leisure time physical activity by the following question: 'How often do you walk outside the home for more than 10 minutes without stopping?' We categorized the responses as 'Rarely', 'Weekly', or 'Daily.'

We defined a participant as having cardiovascular disease if he or she reported using medications for blood pressure or heart disease, or had systolic blood pressure $\geq 140 \mathrm{mmHg}$ and diastolic blood pressure $\geq 90 \mathrm{mmHg}$.

\section{Definition of CKD}

We used the Chronic Kidney Disease Epidemiology Collaboration (CKD-EPI) equation to estimate glomerular filtration rate (eGFR), and the National Kidney Foundation Kidney Disease Outcomes Quality Initiative (NKF/ KDOQI) guidelines to define and stage CKD [11]. We used a sex- specific cut off for albuminuria: $\geq 1.9 \mathrm{mg} /$ $\mathrm{mmol}(17 \mathrm{mg} / \mathrm{g})$ creatinine for men, and $\geq 2.8 \mathrm{mg} / \mathrm{mmol}$ (25 mg/g) creatinine for women. Thus we defined a participant as having CKD if he or she had eGFR below $60 \mathrm{ml} / \mathrm{min} / 1.73 \mathrm{~m}^{2}$ or had albuminuria. We also present prevalence results for albuminuria and CKD using the $\geq 3.4 \mathrm{mg} / \mathrm{mmol}(30 \mathrm{mg} / \mathrm{g})$ creatinine cutoff.

\section{Statistical analyses}

We present continuous data as mean ( \pm standard deviation) and categorical data as proportions, with comparisons via Student's t-test or Pearson's $\chi^{2}$ test, as appropriate. We stratified by sex and performed logistic regression to test the associations among potential covariates including education, wealth status, physical activity, tobacco use, measures of body size (BMI, waist circumference, and waist-to-hip ratio), and presence of insulin resistance or cardiovascular disease. We used generalized linear models to evaluate the association between CKD and the Physical and Mental Health Composite t-scores on the SF-12. We used the New England Medical Center (NEMC) guidelines to score the SF-12 [13]. The primary analysis evaluated these associations using NKF/KDOQI stages of CKD, calculated based on eGFR and/or albuminuria. In multivariable analyses, we adjusted for the residual effects of age and sex, as well as for insulin resistance. Since age and sex are embedded in the eGFR calculation, we performed companion analyses in which we used the reciprocal of serum creatinine as a marker for kidney function. We considered inference tests as significant if the two-tailed p-value was $<005$. We conducted all analyses using SAS, v9.3 (SAS Institute Inc., Cary, NC).

\section{Results}

A total of 357 (89\%) participants provided serum samples and comprise the analytic cohort; 182 (51\%) were men and 175 (49\%) were women. Spot urine albumin and creatinine were assessed among 341 (96\%) participants. As reported previously, men were more likely to be married, employed, and educated at the high school level (Table 1) compared with women. Men also had lower mean BMI, and were more likely to use cigarettes, whereas women more often consumed smokeless tobacco. Two hundred and twelve (59\%) participants had features of insulin resistance, 138 (65\%) of whom had T2DM.

\section{CKD prevalence}

Ninety-four (26\%) participants met the definition of CKD (Figure $1 \mathrm{a}$ and $\mathrm{b}), 45$ men and 49 women. A majority ( $\mathrm{n}=$ $58(62 \%)$ ) had stage one or two CKD (i.e., albuminuria only). Among the 36 participants with stage three or more advanced CKD, 21 (58\%) had associated albuminuria. Overall, there were 79 (22\%) participants with albuminuria, 65 (83\%) of whom had microalbuminuria (Figure 2). Using an albuminuria cutoff of $\geq 3.4 \mathrm{mg} / \mathrm{mmol}$ creatinine, 56 and $72(20 \%)$ participants were classified as having albuminuria and CKD respectively.

\section{Table 1 Characteristics of participants with available} serum creatinine determinations $(n=357)$

\begin{tabular}{|c|c|c|}
\hline & $\begin{array}{l}\text { Men mean } \pm \text { SD } \\
\text { or } N(\%)\end{array}$ & $\begin{array}{c}\text { Women mean } \pm S D \\
\text { or } N(\%)\end{array}$ \\
\hline \multicolumn{3}{|l|}{ Demographics } \\
\hline Age (in years) & $49 \cdot 4 \pm 12 \cdot 1$ & $496 \pm 13 \cdot 4$ \\
\hline Married* & $170(93)$ & $125(71)$ \\
\hline $\begin{array}{l}\text { High school education } \\
\text { or more* }\end{array}$ & $96(53)$ & $53(30)$ \\
\hline Employed* & $139(76)$ & $125(71)$ \\
\hline Wealth index $>3+$ & $33(18)$ & $34(19)$ \\
\hline Residence in Dhaka (in years)* & $30 \cdot 0 \pm 16 \cdot 2$ & $33 \cdot 8 \pm 15 \cdot 1$ \\
\hline \multicolumn{3}{|l|}{ Clinical characteristics } \\
\hline \multicolumn{3}{|l|}{ BMI $\left(\mathrm{kg} / \mathrm{m}^{2}\right)$ categories } \\
\hline$<25$ & 92 & 40 \\
\hline 25 to $<30$ & 82 & 71 \\
\hline$\geq 30$ & 51 & 21 \\
\hline Smoker (current or past)* & $107(59)$ & $5(3)$ \\
\hline Smokeless tobacco user* & $30(17)$ & $45(26)$ \\
\hline Self-reported hypertension* & $57(31)$ & $76(43)$ \\
\hline $\begin{array}{l}\text { Measured systolic blood } \\
\text { pressure }\end{array}$ & $1184 \pm 157$ & $1178 \pm 166$ \\
\hline $\begin{array}{l}\text { Measured diastolic blood } \\
\text { pressure }\end{array}$ & $74 \cdot 6 \pm 11 \cdot 2$ & $74 \cdot 5 \pm 13 \cdot 3$ \\
\hline
\end{tabular}

*represents significant difference at the $p<0.05$ level. $†$ A wealth index above 3 indicates owning 3 or more of: apartment/house, car, power source, or air conditioner. Abbreviations: BMI-Quételet's (body mass) index,

SD-standard deviation. 


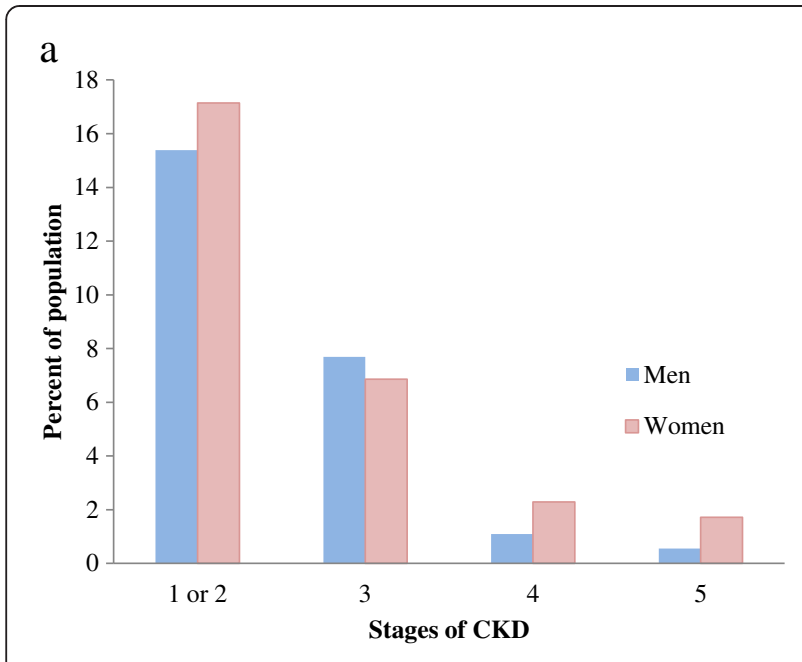

b

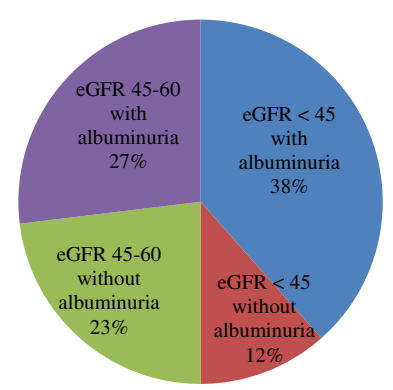

Figure 1 Stages of CKD with details on albuminuria for stage three CKD. a. Stages of CKD. Overall 94 (27\%) of participants had CKD. Stage one or two CKD (albuminuria with eGFR $>90$ or between $60-90 \mathrm{ml} / \mathrm{min} / 1.73 \mathrm{~m}^{2}$ ) was present in 58 (62\%). Stage five CKD was present in 4 (4\%). Abbreviations: CKD-Chronic kidney disease, eGFR-estimated glomerular filtration rate. $\mathbf{b}$. Characterizing stage three CKD. Among participants with stage three CKD $(n=26,28 \%), 6(23 \%)$ had an isolated reduction in eGFR between $45-59 \mathrm{ml} / \mathrm{min} / 1.73 \mathrm{~m}^{2}$; all others had either stage $3 b$ CKD or accompanying albuminuria. Abbreviations: CKD-chronic kidney disease; eGFR-estimated glomerular filtration rate.

\section{Clinical correlates of CKD in Bangladesh}

On unadjusted analyses, education level was not associated with the presence of CKD among men, although among women, those who had received fewer than 5 years of education experienced higher odds of CKD compared with women educated at the University level or beyond (Odds ratio [OR] 36, 95\% Confidence Interval [CI] 14 to 94). Wealth status was not associated with CKD among women or men. Since smokers in our sample were predominantly (96\%) men, we tested the association between smoking and CKD among men only and found no association. Use of smokeless (chewable) tobacco was associated with higher odds of CKD among women (OR 28, 95\% CI 14 to 57).

Larger waist circumference and waist-to-hip ratio (using International Diabetes Foundation criteria) [12], BMI, and leisure-time physical activity were not significantly associated with CKD.

\section{CKD and insulin resistance}

A majority of participants with CKD also had features of insulin resistance (Figure 3). The unadjusted odds of CKD among participants with insulin resistance were 3.6 fold higher (95\% CI $2 \cdot 1$ to 64 ) than among participants without insulin resistance. Upon stratification by sex, the odds of CKD among men with insulin resistance were 3.1 (95\% CI 1.5 to 64 ) and among women were 4.7 (95\% CI 19 to 119 ). Cardiovascular disease was also evident in more than two-thirds $(n=64,68 \%)$ of participants with CKD. The measured average blood pressure of a participant with CKD was $125 / 78 \mathrm{mmHg}$, approximately $10 / 5$ points higher than in participants without CKD ( $p<0.001$ and 0.005, for systolic and diastolic pressure, respectively).

\section{CKD and HRQoL}

Mean t-scores on the SF-12 NEMC Physical Health Composite t-Score (PCS) were lower among participants with CKD (Table 2). There was no significant difference in mean t-scores on the SF-12 Mental Health Composite Score (MCS), although the mean MCS for participants with stage five CKD was significantly lower than for all other stages. Multivariable analyses adjusting for the residual effects of age and gender as well as for insulin resistance showed that mean scores on PCS were significantly lower among participants with stage three or more advanced CKD.

On companion analyses using the reciprocal of serum creatinine as a main exposure of interest (as opposed to eGFR), we found that the PCS was inversely correlated with serum creatinine and age, and was lower among women compared with men.

\section{Discussion}

Among randomly sampled adults living in a middleincome neighborhood of Bangladesh, approximately one in five were found to have CKD. Nearly four of five participants with CKD had features of insulin resistance, which was the predominant risk factor linked with CKD in our study. In contrast to data from the U.S. and other countries where stage three CKD predominates [14-16], we found that stage one or two CKD (albuminuria only) was most common. The high prevalence of insulin resistance and associated albuminuria among our participants with CKD implies that they are at high risk for progression of CKD and for experiencing a cardiovascular event.

No prior data exist on prevalence of CKD in Bangladesh. Two studies from New Delhi, India have used variable definitions of CKD. A study of 4,712 adults living in South Delhi found that $0.8 \%$ had serum creatinine above 159 umol/L (1.8 $\mathrm{mg} / \mathrm{dL})$ [5]; the comparable percentage in our 


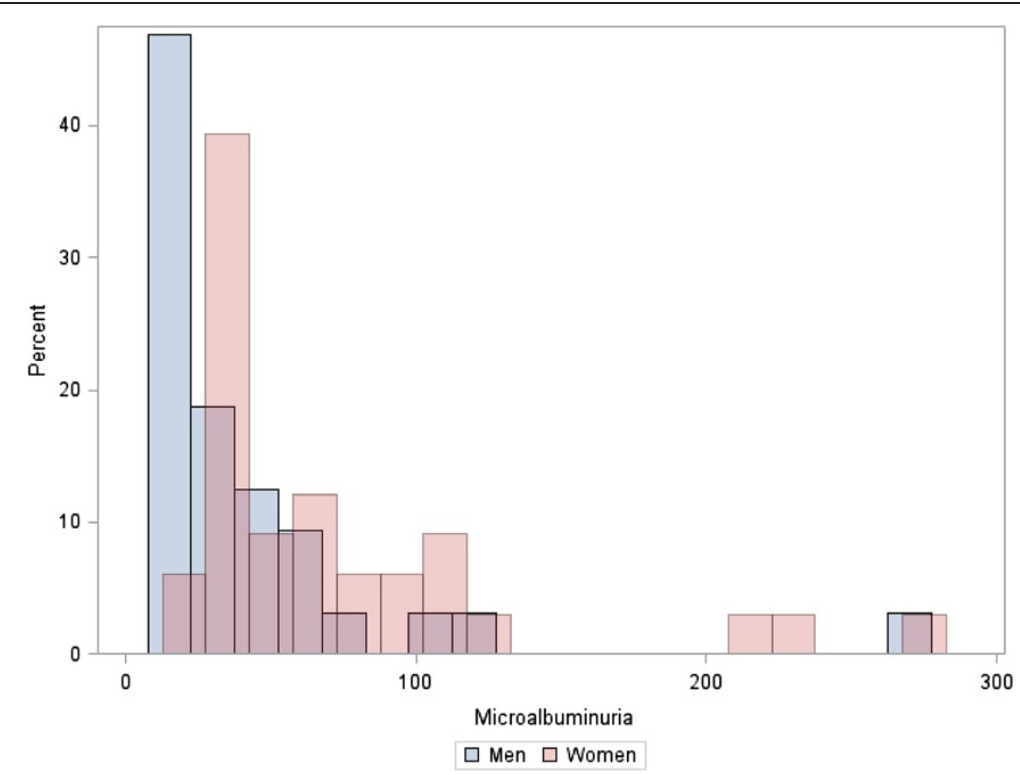

Figure 2 Distribution of microalbuminuria in men and women. Microalbuminuria was defined as $1.9-34 \mathrm{mg} / \mathrm{mmol}(17-300 \mathrm{mg} / \mathrm{g})$ creatinine for men and $(2.8-34 \mathrm{mg} / \mathrm{mmol}(25-300 \mathrm{mg} / \mathrm{g})$ creatinine for women. There were a total of $79(22 \%)$ cases of albuminuria, 65 ( $83 \%)$ of which were in the microalbuminuria range.

study was $3 \%$. Another study of 5,252 adults also based in Delhi reported a prevalence of reduced eGFR (below $60 \mathrm{ml} / \mathrm{min} / 1.73 \mathrm{~m}^{2}$ ) equal to $4 \%$ [6]; the comparable percentage in our study was $10 \%$. The Thai-Global Screening and Early Evaluation of Kidney Disease (SEEK) study sampled 3,459 from 10 provinces. Both albuminuria and serum creatinine were evaluated and the standard NKF/ KDOQI definition was applied; $17 \%$ of surveyed participants met criteria for CKD [17]. As reference, the most recent estimates of CKD prevalence from Norway [18], Spain [15], U.S. [14], and Japan [16] are between 10 and $13 \%$ of the adult population.

We hypothesize three reasons for the higher prevalence of CKD noted in our study. First, we surveyed adults over 30 years old, whereas Thai-SEEK and NHANES enrolled

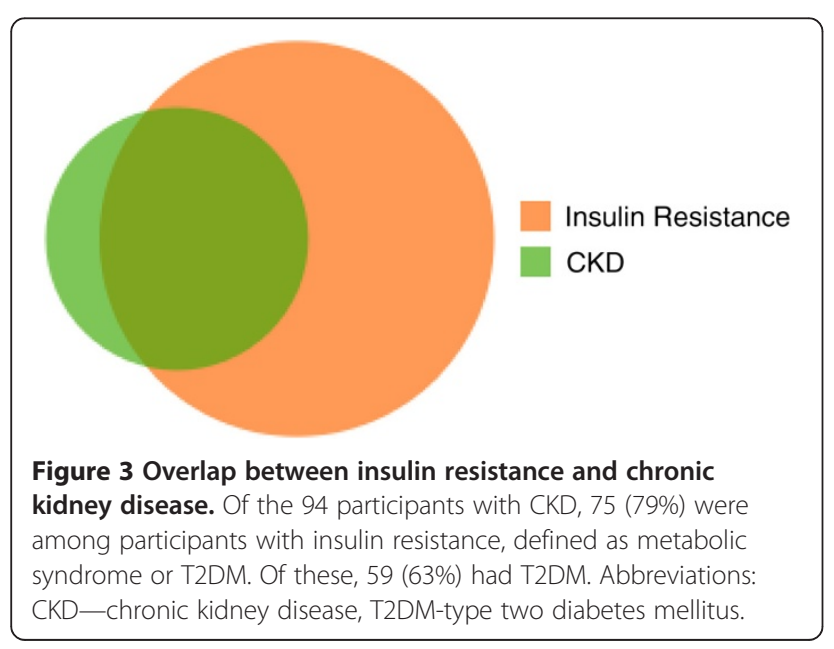

adults over 18 and 20 years old, respectively. Second, by surveying a well-established neighborhood of a major urban hub, we are sampling from a population at higher risk, not representative of the risk experienced by people living in rural areas or poorer persons. Migrating to an

Table 2 SF-12 scores among participants with chronic kidney disease

\begin{tabular}{lcc}
\hline & $\begin{array}{c}\text { PCS } \\
\text { Mean } \pm \text { SE }\end{array}$ & $\begin{array}{c}\text { MCS } \\
\text { Mean } \pm \text { SE }\end{array}$ \\
\hline Unadjusted model & & \\
No chronic kidney disease (ref) & $40.2 \pm 0.6$ & $41.1 \pm 0.7$ \\
Chronic kidney disease $(\mathrm{n}=94)$ & & \\
Stage 1 or $2(\mathrm{n}=58)$ & $\mathbf{3 8 . 6} \pm \mathbf{1 . 3}$ & $43.0 \pm 1.2$ \\
Stage $3(\mathrm{n}=26)$ & $\mathbf{3 4 . 9} \pm \mathbf{2 . 0}$ & $45.6 \pm 1.7$ \\
Stage $4(\mathrm{n}=6)$ & $\mathbf{2 9 . 2} \pm \mathbf{2 . 0}$ & $32.9 \pm 6.2$ \\
Stage 5 ( $\mathrm{n}=4)$ & $\mathbf{2 7 . 6} \pm \mathbf{2 . 2}$ & $\mathbf{2 3 . 3} \pm \mathbf{4 . 2}$ \\
Adjusted model* & & \\
No chronic kidney disease $(\mathrm{ref})$ & & \\
Chronic kidney disease & $39.8 \pm 0.5$ & $40.9 \pm 0.7$ \\
Stage 1 or 2 & & \\
Stage 3 & $39.1 \pm 1.1$ & $43.3 \pm 1.4$ \\
Stage 4 & $\mathbf{3 6 . 6} \pm \mathbf{1 . 8}$ & Up \\
Stage 5 & $\mathbf{3 2 . 2} \pm \mathbf{3 . 5}$ & $34.6 \pm 4.6$ \\
\hline
\end{tabular}

Values in bold are significantly different from the referent category. *Adjusted for insulin resistance, and residual effects of age and gender. Stages are based on the National Kidney Foundation/Kidney Disease Outcomes Quality Initiative definition of chronic kidney disease. Abbreviations: PCS: New England Medical Center Physical Health Score, MCS: Mental Health Score, SE: standard error. 
urban area was associated with an approximately 1.5-fold increase in odds of a sedentary lifestyle, three-fold increase in odds of obesity, and two-fold increase in odds of T2DM among men working in factories in India, compared with their rural counterparts [3]. In low-income countries, the prevalence of obesity increases with increasing wealth, although this trend may soon arrest or reverse as prices of fruits and vegetables climb while processed foods become cheaper and more easily accessible [19].

A majority of participants who were identified as having CKD had stage one or two CKD (i.e., albuminuria only). As we did not repeat assessment of albuminuria, we do not have population-specific data on persistent albuminuria. However extrapolating data from NHANES on spot versus persistent albuminuria, $51 \%$ of participants with microalbuminuria and stage one CKD and $75 \%$ of participants with microalbuminuria and stage two CKD would be estimated to have persistent microalbuminuria, yielding an adjusted (approximate) CKD prevalence equal to 19\%, rather than $26 \%$. Thus, the prevalence of CKD in the urban Bangladeshi population would still be higher than that in the U.S., Europe, and Japan. Strikingly, we identified four persons (1\%) with stage five CKD (eGFR below $15 \mathrm{ml} / \mathrm{min} / 1.73 \mathrm{~m}^{2}$ ). If we extrapolate only to the $>30$ year old population of Mohammedpur, a stage five CKD prevalence of $1 \%$ would imply that roughly 1,800 persons would be at risk for imminent kidney failure in a district that represents $0.3 \%$ of the Bangladeshi population. Even these 1,800 persons represent an immense burden in a country where kidney transplants are performed in only one and dialysis in just four of 13 government hospitals [20].

Aside from the need for transplantation or dialysis, persons with CKD (especially those with albuminuria) experience an increased risk for cardiovascular disease and mortality. Microalbuminuria, found in $70 \%$ of our participants with CKD, has been linked to a 30 to $80 \%$ increase in the risk for all-cause and cardiovascular mortality; overt proteinuria, found in $15 \%$ of our participants with CKD, can double or triple these risks [21]. The majority of our participants with CKD also had T2DM, which increases risk for all-cause and cardiovascular mortality 20 to $90 \%$, relative to persons with CKD who do not have T2DM [1].

In Bangladesh, care for chronic illnesses is not yet in line with the care for infectious diseases; much of the public sector health budget is devoted to treating episodic illnesses such as diarrhea or respiratory illness. No national guidelines exist for primary health care screening for chronic illnesses such as CKD. Even among patients identified to have a chronic illness, cost of treatment is a major burden: while the government subsidizes some of the care (e.g., the doctor's visit) in the public sector, patients may still pay for other aspects (e.g., medications). Some subsidies are available from social welfare organizations. Those who can afford it do seek private care, and a negligible percent goes abroad for better treatment.

We also found that moderate to advanced CKD was associated with a decrement in self-reported physical health. While few studies have explored HRQoL in patients with non-dialysis requiring CKD, the associated decrement in self-reported physical health seen among participants with moderate to advanced CKD in this study is considered to be clinically meaningful [22]. Thus, if the burden of CKD is not lessened in the coming decades, we can anticipate higher rates of cardiovascular disease, frailty, and debility in the urban Bangladeshi population, even in late middle and early older age.

Our study has several strengths. We adopted a random sampling procedure in this community-based survey. Trained and experienced nurses and research assistants used standardized protocols for physical measurements. Anticipating low rates of awareness of chronic diseases, we employed laboratory measurement to diagnose T2DM, metabolic syndrome, and CKD. Ours is one of few studies in the region to include an assessment of albuminuria and HRQoL.

However, given our modest sample size and that our survey was limited to a particular urban neighborhood, we cannot extrapolate our findings to all of Bangladesh. Our modest sample size also limited our assessment of the correlates of CKD. Ideally, repeat measures of serum creatinine and urinary albumin excretion might allow us to be more precise in our prevalence estimates.

\section{Conclusions}

Our study points to an alarmingly high rate of CKDroughly one in five-among urban middle-income Bangladeshis. The kidney disease we detected will pose a significant clinical burden to this resource-poor setting with limited healthcare infrastructure and very limited capacity to manage advanced and end-stage disease. Additional studies are required to define CKD prevalence, incidence rates, and region- and population-specific risk factors in low- and middle-income countries around the globe, as well as an urgent need to design low-cost programs to detect and manage CKD in Bangladesh and other countries in South Asia.

\section{Abbreviations}

CKD: Chronic kidney disease; CKD-EPI: Chronic kidney disease epidemiology collaboration; Cl: Confidence interval; eGFR: Estimated glomerular filtration rate; HRQOL: Health-related quality of life; ICDDR,B: International center for diarrheal disease research, Bangladesh; SF-12: Medical outcomes study short form-12; MCS: Mental health composite t-score; NKF/KDOQl: National kidney foundation/kidney disease outcomes quality initiative; NHANES: National health and nutrition examination survey; NEMC: New England medical center; OR: Odds ratio; PCS: Physical health composite t-score; BMI: Quételet's (body mass) index; SEEK: Screening and early evaluation of kidney disease; T2DM: Type 2 diabetes mellitus. 


\section{Competing interests}

The authors have no conflicts of interest to declare. This work has not been published elsewhere

\section{Authors' contributions}

Dr. SA was helped with survey design, and data collection and analysis, and performed the manuscript write-up. Dr. AK led survey design, and data collection. Drs JS, TA, and DSA helped with survey design, and data collection and analysis, and provided editorial input for manuscript write-up. Drs MB, MRC, and GMC helped secure funding for data collection, advised survey design, and provided editorial input for manuscript write-up. All authors read and approved the final manuscript.

\section{Acknowledgements}

Dr. Anand was supported by the National Institute of Health Research Training Grant R25 TW009338 funded by the Fogarty International Center. Dr. Chertow was supported by K24 DK 085446.

\section{Author details}

'Stanford University School of Medicine, 291 Campus Dr, Stanford, CA 94305. USA. ${ }^{2}$ International Center for Diarrheal Disease Research, 68 Shaheed Tajuddin Ahmed Sharani, Mohakhali, Dhaka 1212, Bangladesh. ${ }^{3}$ Division of Nephrology, Stanford University School of Medicine, 777 Welch Road, Suite DE, Palo Alto, CA 94304, USA. ${ }^{4}$ Centre of Clinical Epidemiology and Biostatistics, School of Medicine and Public Health, University of Newcastle, Australia

Received: 21 August 2013 Accepted: 6 February 2014

Published: 20 February 2014

\section{References}

1. Barsoum RS: Chronic kidney disease in the developing world. N Engl J Med 2006, 354(10):997-999.

2. Srinath Reddy K, Shah B, Varghese C, Ramadoss A: Responding to the threat of chronic diseases in India. Lancet 2005, 366(9498):1744-1749.

3. Ebrahim S, Kinra S, Bowen L, Andersen E, Ben-Shlomo Y, Lyngdoh T, Ramakrishnan L, Ahuja RC, Joshi P, Das SM, Mohan M, Davey Smith G, Prabhkaran D, Reddy KS: The effect of rural-to-urban migration on obesity and diabetes in India: a cross-sectional study. PLoS Med 2010, 7(4):e1000268

4. International Diabetes Federation: Diabetes Atlas. Belgium: IDF; 2011.

5. Agarwal SK, Dash SC, Irshad M, Raju S, Singh R, Pandey RM: Prevalence of chronic renal failure in adults in Delhi, India. Nephrol Dial Transplant 2005, 20(8):1638-1642

6. Singh NP, Ingle GK, Saini VK, Jami A, Beniwal P, Lal M, Meena GS: Prevalence of low glomerular filtration rate, proteinuria and associated risk factors in North India using Cockcroft-Gault and modification of diet in renal disease equation: an observational, cross-sectional study. BMC Nephrol 2009, 10:4

7. Jafar TH, Schmid CH, Levey AS: Serum creatinine as marker of kidney function in South Asians: a study of reduced GFR in adults in Pakistan. J Am Soc Nephrol 2005, 16(5):1413-1419.

8. Caballero B: A nutrition paradox-underweight and obesity in developing countries. N Engl J Med 2005, 352(15):1514-1516.

9. Bangladesh Bureau of Statistics: Bangladesh Data Sheet 2009. 2011. [cited 2012 December 20]; Available from: http://www.bbs.gov.bd/.

10. World Health Organization: WHO steps manual: guide to physical measurements 2013. World Health Organization, 2011 [cited 2012 December 24]; Available from: http://www.who.int/chp/steps/manual/en/.

11. National Kidney Foundation, T: KDOQI Clinical practice guidelines for chronic kidney disease: evaluation, classification, and stratification. 2002. [cited 2013 March]; Available from: http://www.kidney.org/professionals/ KDOQI/guidelines_ckd/.

12. Alberti KG, Zimmet $P$, Shaw J: Metabolic syndrome-a new world-wide definition. A consensus statement from the International Diabetes Federation. Diabet Med 2006, 23(5):469-480.

13. Ron Hays CDS, Karen S, Dixon WJ: A microcomputer program (sas.exe) that generates SAS code for scoring the SF-36 Health Survey. RAND 1996. DOI: http://www.rand.org/content/dam/rand/pubs/drafts/2007/DRU1437. pdf.
14. Coresh J, Selvin E, Stevens LA, Manzi J, Kusek JW, Eggers P, Van Lente F, Levey AS: Prevalence of chronic kidney disease in the United States. JAMA 2007, 298(17):2038-2047.

15. Otero A, de Francisco A, Gayoso P, Garcia F, Group ES: Prevalence of chronic renal disease in Spain: results of the EPIRCE study. Nefrologia 2010, 30(1):78-86.

16. Imai E, Horio M, Watanabe $T$, Iseki $K$, Yamagata $K$, Hara S, Ura N, Kiyohara $Y$, Moriyama T, Ando Y, Fujimoto S, Konta T, Yokoyama H, Makino H, Hishida A, Matsuo S: Prevalence of chronic kidney disease in the Japanese general population. Clin Exp Nephrol 2009, 13(6):621-630.

17. Ingsathit A, Thakkinstian A, Chaiprasert A, Sangthawan $P$, Gojaseni $P$, Kiattisunthorn K, Ongaiyooth L, Vanavanan S, Sirivongs D, Thirakhupt $P$ Mittal B, Singh AK: Prevalence and risk factors of chronic kidney disease in the Thai adult population: Thai SEEK study. Nephrol Dial Transplant 2010, 25(5):1567-1575.

18. Hallan SI, Coresh J, Astor BC, Asberg A, Powe NR, Romundstad S, Hallan HA, Lydersen S, Holmen J: International comparison of the relationship of chronic kidney disease prevalence and ESRD risk. J Am Soc Nephrol 2006 17(8):2275-2284

19. McLaren L: Socioeconomic status and obesity. Epidemiol Rev 2007, 29:29-48.

20. Ur Rashid $\mathrm{H}$ : Health delivery system for renal disease care in bangladesh. Saudi J Kidney Dis Transp/ 2004, 15(2):185-189.

21. Fox CS, Matsushita K, Woodward M, Bilo HJ, Chalmers J, Heerspink HJ, Lee BJ, Perkins RM, Rossing P, Sairenchi T, Tonelli M, Vassaloti JA, Yamagishi K, de Jong PE, Wen CP, Nelson RG: Associations of kidney disease measures with mortality and end-stage renal disease in individuals with and without diabetes: a meta-analysis. Lancet 2012, 380(9854):1662-1673.

22. Gorodetskaya I, Zenios S, McCulloch CE, Bostrom A, Hsu CY, Bindman AB, Go AS, Chertow GM: Health-related quality of life and estimates of utility in chronic kidney disease. Kidney Int 2005, 68(6):2801-2808.

doi:10.1186/1744-8603-10-9

Cite this article as: Anand et al.: High prevalence of chronic kidney

disease in a community survey of urban Bangladeshis: a cross-sectional study. Globalization and Health 2014 10:9.

\section{Submit your next manuscript to BioMed Central and take full advantage of:}

- Convenient online submission

- Thorough peer review

- No space constraints or color figure charges

- Immediate publication on acceptance

- Inclusion in PubMed, CAS, Scopus and Google Scholar

- Research which is freely available for redistribution 